\title{
Nucleon in a periodic magnetic field
}

\author{
Andria Agadjanov ${ }^{a}$, Ulf-G. Meißner ${ }^{a, b}$ and Akaki Rusetsky ${ }^{a}$ \\ ${ }^{a}$ Helmholtz-Institut für Strahlen- und Kernphysik (Theorie) and Bethe Center for Theoretical Physics, \\ Universität Bonn, D-53115 Bonn, Germany and \\ ${ }^{b}$ Institute for Advanced Simulation (IAS-4), Institut für Kernphysik (IKP-3) and Jülich Center for Hadron Physics,
} Forschungszentrum Jülich, D-52425 Jülich, Germany

(Dated: December 17, 2018)

\begin{abstract}
The energy shift of a nucleon in a static periodic magnetic field is evaluated at second order in the external field strength in perturbation theory. It is shown that the measurement of this energy shift on the lattice allows one to determine the unknown subtraction function in the forward doubly virtual Compton scattering amplitude. The limits of applicability of the obtained formula for the energy shift are discussed.
\end{abstract}

\section{INTRODUCTION}

The doubly virtual Compton scattering process has several important phenomenological implications at low energies. The analysis of the proton-neutron mass difference $\frac{1}{3}\left[\begin{array}{l}3 \\ 6\end{array}\right]$ relies on the knowledge of the relevant spinindependent invariant amplitudes $T_{1}$ and $T_{2}$. The same amplitudes appear in the study of the Lamb shift in the muonic hydrogen (see, e.g., Refs. 7-9]).

The experimental data on the structure functions completely determine the amplitude $T_{2}$. However, they do not fix the subtraction function $S_{1}$ in the dispersion relation for the amplitude $T_{1}$. This function depends on the photon virtuality $q^{2}$. The low-energy theorem establishes a relation between the value of the $S_{1}\left(q^{2}\right)$ at $q^{2}=0$ and the magnetic polarizability of the nucleon. Further, the asymptotic behavior of this function for large spacelike $q^{2}$ is fixed by the operator product expansion in QCD [6, 10, 11]. The behavior of the function at intermediate values of $q^{2}$ is, however, completely arbitrary. Earlier calculations of the forward Compton scattering amplitude within chiral effective field theories at low photon virtualities have been carried out in Refs. [12 15]. The latest calculations of the quantity $S_{1}$ are contained in Refs. [7, 9, 16 -19]. The convergence of the chiral expansion is, however, questionable even at very low $q^{2}$. Further, several authors have used phenomenological parametrizations of the function $S_{1}$ in their calculations [5, 6, 8]. Unfortunately, this introduces a systematic uncertainty in the calculated observables that is very hard to control.

An interesting possibility to determine the subtraction function $S_{1}$ was discussed in Refs. [3, 4]. If the forward scattering amplitude does not contain any so-called fixed pole (this issue is related to the so-called Reggeon dominance hypothesis), then $S_{1}\left(q^{2}\right)$ for all $q^{2}<0$ is uniquely

\footnotetext{
${ }^{1}$ Note that, recently, a substantial progress has been achieved in the direct evaluation of this difference on the lattice [1, 2]. A comparison of different approaches provides constaints on the behavior of the Compton amplitudes.
}

determined by the dispersion integral over the electroproduction cross sections in the physical region. Therefore, if one could calculate the function $S_{1}\left(q^{2}\right)$ directly and compare with the result obtained by using the Reggeon dominance hypothesis, in principle one would be able to answer a question, whether a fixed pole is present in the forward Compton amplitude or not (for the recent phenomenological evaluation of $S_{1}$, see Refs. [4, 20, 21]). For instance, note that the universality hypothesis, stated in Ref. 22], does not exclude the presence of a fixed pole in $T_{1}$. Further, a calculation of $S_{1}\left(q^{2}\right)$ would allow one to evaluate the proton-neutron electromagnetic mass shift and the two-photon exchange contribution to the muonic hydrogen Lamb shift in a manner devoid of any model dependence. Hence, there is strong interest in a direct calculation of the function $S_{1}\left(q^{2}\right)$.

At present, lattice QCD is the only first-principle approach capable of handling the above problem. There are two ways to determine $S_{1}$. In the first method, one directly calculates the four-point function that describes Compton scattering. This is a straightforward but computationally very demanding task. Until now, this approach has been used in the computation of light-bylight scattering [23], and also in the study of the longdistance effects in rare kaon decays [24, 25]. An alternative method is based on the observation that the Compton scattering amplitude can be inferred from the behavior of the nucleon two-point function in the presence of a weak external electromagnetic field. In recent years, the external field method has become a powerful tool to study the electromagnetic properties of the nucleon and light nuclei. In particular, it has been used for the case of a constant and uniform magnetic field [26 28]. Such a field configuration allows one to determine the magnetic moments and magnetic polarizabilities through the extracted energy shift induced by the magnetic field. By applying nonuniform and time-dependent fields, one can determine spin polarizabilities as well (see, e.g., Refs. $[29,[30]$ ). Hence, the external field approach for the calculation of $S_{1}$ should be feasible in practice.

In this paper, we demonstrate that measuring the energy shift of a single nucleon state in a static periodic 
magnetic field on the lattice enables one to determine the function $S_{1}\left(q^{2}\right)$ at nonzero values of $q^{2}$. Unlike a constant magnetic field, which generates the harmonic oscillator potential and Landau levels, in the present case the spectrum and the eigenstates of the Hamiltonian can not be obtained analytically. Nevertheless, the energy is still conserved in the static magnetic field and, as long as the potential remains "small," perturbation theory can be applied to the free energy spectrum (there is a similar approach in solid-state physics, which is called the nearly free electron model, and more generally, the empty lattice approximation [31]). We show that, at second order in the magnetic field strength, the energy shift of the one-nucleon ground state is proportional to the quantity $S_{1}\left(q^{2}\right)$. The limits of applicability of this perturbative expression are also discussed.

\section{COMPTON SCATTERING}

Let us start with the basic definitions. The Compton scattering amplitude is given by

$$
T^{\mu \nu}\left(p^{\prime}, s^{\prime} ; p, s ; q\right)=\frac{i}{2} \int d^{4} x e^{i q \cdot x}\left\langle p^{\prime}, s^{\prime}\left|T j^{\mu}(x) j^{\nu}(0)\right| p, s\right\rangle \text {. }
$$

Here, $j^{\mu}(x)$ denotes the electromagnetic current, $q$ is the four-momentum of the final photon, $p\left(p^{\prime}\right)$ and $s\left(s^{\prime}\right)$ are the four-momenta and spins of the initial (final) nucleon, respectively. Considering forward scattering $p^{\prime}=p$ and performing the spin-averaging in Eq. (1), one arrives at

$$
T^{\mu \nu}(p, q)=\frac{1}{2} \sum_{s} T^{\mu \nu}(p, s ; p, s ; q) .
$$

The tensor $T^{\mu \nu}(p, q)$ is related to the invariant amplitudes $T_{1}, T_{2}$ through the expression (see, e.g., Refs. [4, 32, 33]):

$$
T^{\mu \nu}(p, q)=T_{1}\left(\nu, q^{2}\right) K_{1}^{\mu \nu}+T_{2}\left(\nu, q^{2}\right) K_{2}^{\mu \nu},
$$

where the kinematic structures $K_{1}^{\mu \nu}, K_{2}^{\mu \nu}$ read

$$
\begin{aligned}
& K_{1}^{\mu \nu}=q^{\mu} q^{\nu}-g^{\mu \nu} q^{2}, \\
& K_{2}^{\mu \nu}=\frac{1}{m^{2}}\left\{\left(p^{\mu} q^{\nu}+p^{\nu} q^{\mu}\right) p \cdot q-g^{\mu \nu}(p \cdot q)^{2}-p^{\mu} p^{\nu} q^{2}\right\} .
\end{aligned}
$$

Here, $m$ is the nucleon mass and $\nu \equiv p \cdot q / m$. The subtraction function $S_{1}$ is defined as

$$
S_{1}\left(q^{2}\right)=T_{1}\left(0, q^{2}\right) .
$$

The quantity $S_{1}\left(q^{2}\right)$ can be split into the elastic and inelastic parts (see, e.g., Ref. [4]). The elastic part is singular at $q^{2} \rightarrow 0$, whereas the inelastic part is regular and is related to the nucleon magnetic polarizability at $q^{2}=0$. Note that the quantity $S_{1}\left(q^{2}\right)$ is real, because in this kinematical region there are no multi-particle singularities. Indeed, introducing the Mandelstam variable $s=(p+q)^{2}=m^{2}+2 m \nu+q^{2}$, it is immediately seen that, for $\nu=0$ and $q^{2}<0$, we have $s<m^{2}$, meaning that one is below the inelastic threshold.

It is well known that the Compton tensor given in Eq. (11) can be obtained by expanding the two-point function of a nucleon in an external electromagnetic field to second order. Introducing the notation $A_{\mu}(x)$ for the external potential, it is actually seen that the nucleon propagator can be expanded as follows:

$$
\begin{aligned}
\langle 0|T \Psi(x) \bar{\Psi}(y)| 0\rangle_{A} & =\langle 0|T \Psi(x) \bar{\Psi}(y)| 0\rangle_{0}+\frac{i}{1 !} \int d^{4} z A_{\mu}(z)\left\langle 0\left|T \Psi(x) \bar{\Psi}(y) j^{\mu}(z)\right| 0\right\rangle_{0} \\
& +\frac{i^{2}}{2 !} \int d^{4} z d^{4} v A_{\mu}(z) A_{\nu}(v)\left\langle 0\left|T \Psi(x) \bar{\Psi}(y) j^{\mu}(z) j^{\nu}(v)\right| 0\right\rangle_{0}+\cdots
\end{aligned}
$$

Here, $\Psi(x)$ denotes the (composite) nucleon field operator in QCD and the subscript "0" refers to the quantities evaluated in QCD without any external field. Note that Eq. (6) is written down for connected matrix elements (the subscript "conn" is omitted everywhere for brevity). Further, performing the Fourier transform in Eq. (6), amputating the external nucleon legs, and putting external nucleons on the mass shell, we see that the nucleon electromagnetic vertex $\left\langle p^{\prime}, s^{\prime}\left|j^{\mu}(0)\right| p, s\right\rangle$ emerges at order $A$. At order $A^{2}$, as already mentioned, the scattering amplitude given in Eq. (11) is obtained from the matrix element $\left\langle 0\left|T \Psi(x) \bar{\Psi}(y) j^{\mu}(z) j^{\nu}(v)\right| 0\right\rangle_{0}$, and so on.

On the other hand, since one is below the inelastic threshold, one may describe the nucleon two-point function within the nonrelativistic effective field theory as well, matching the couplings of the effective Lagrangian to the pertinent expressions in QCD. The advantage of this approach will become apparent, when the energy spectrum of a system in a finite box will be considered, as in the nonrelativistic effective theory, the energy levels are obtained by merely solving the Schrödinger equation.

In the present work we consider the matching and the subsequent calculation of the energy shift in a very condensed manner. A detailed treatment of these issues, as well as a thorough study of the finite-volume spectrum of the Hamiltonian with periodic potentials wil be the 
subject of a separate publication [34]. In brief, the procedure looks as follows. At the first stage, the matching of the relativistic and nonrelativistic theories is carried out in the infinite volume. The matching ensures that the on-shell coefficients in the expansion of the two-point function up to and including $O\left(A^{2}\right)$ are reproduced in the nonrelativistic theory. At the next step, one uses the nonrelativistic Hamiltonian, whose couplings are fixed through the matching, to calculate the energy levels in a finite volume. Note that this procedure is self-consistent, since the couplings of the effective Hamiltonian encode solely the short-range physics that does not get altered by placing the system in a large box.

Above, we have already written down the expansion of the nucleon two-point function in the external field in QCD. Now, we want to do the same in the effective theory. The corresponding Lagrangian, which describes the gauge-invariant interaction of the nucleon with an external electromagnetic field, has the following general form:

$$
\mathcal{L}_{\text {eff }}=\mathcal{L}_{0}+\mathcal{L}_{1}+\mathcal{L}_{2}+\cdots
$$

Here, $\mathcal{L}_{0}$ is the free nucleon Lagrangian (we remind the reader that we are below the inelastic cuts)

$$
\mathcal{L}_{0}=\psi^{\dagger} 2 W\left(i \partial_{t}-W\right) \psi, \quad W=\sqrt{m^{2}-\nabla^{2}} .
$$

In the above expression, $\psi(x)$ is a two-component field that describes the nonrelativistic nucleon. It is seen that the relativistic dispersion relation for the energy of the free nucleon with the three-momentum $\mathbf{p}, w(\mathbf{p})=$ $\sqrt{m^{2}+\mathbf{p}^{2}}$, is satisfied. Also, the factor $2 W$ ensures that the one-particle states have the relativistic normalization (see, e.g., Refs. 35, 36]).

Further, $\mathcal{L}_{1}$ is linear in the external field $A_{\mu}, \mathcal{L}_{2}$ is quadratic in $A_{\mu}$, and so on. Note that $\mathcal{L}_{1}$ and $\mathcal{L}_{2}$ should contain an infinite set of operators with an arbitrary number of space derivatives, which act on the fermion and external fields. This differs from the situation for vanishing $q^{2}$ (the pertinent Lagrangian is given, e.g., in Ref. [29]). However, as we shall see, the infinite number of terms will effectively sum up in a single function.

Applying the equation of motion to eliminate the time derivatives, and performing partial integration, $\mathcal{L}_{1}$ and $\mathcal{L}_{2}$ can be brought into the form

$$
\begin{aligned}
\mathcal{L}_{1} & =\sum_{m, n=0} A_{\mu}(x)\left[\partial_{i_{1}} \ldots \partial_{i_{n}} \psi_{s^{\prime}}^{\dagger}(x)\right] \Gamma_{s^{\prime} s}^{i_{1} \ldots i_{n}, j_{1} \ldots j_{m}, \mu}\left[\partial_{j_{1}} \ldots \partial_{j_{m}} \psi_{s}(x)\right] \\
\mathcal{L}_{2} & =\sum_{l, m, n=0} A_{\nu}(x)\left[\partial_{\mu_{1}} \ldots \partial_{\mu_{l}} A_{\mu}(x)\right]\left[\partial_{i_{1}} \ldots \partial_{i_{n}} \psi_{s^{\prime}}^{\dagger}(x)\right] \Pi_{s^{\prime} s}^{i_{1} \ldots i_{n}, j_{1} \ldots j_{m}, \mu_{1} \ldots \mu_{l}, \mu \nu}\left[\partial_{j_{1}} \ldots \partial_{j_{m}} \psi_{s}(x)\right]
\end{aligned}
$$

where $\Gamma_{s^{\prime} s}^{i_{1} \ldots i_{n}, j_{1} \ldots j_{m}, \mu}$ and $\Pi_{s^{\prime} s}^{i_{1} \ldots i_{n}, j_{1} \ldots j_{m}, \mu_{1} \ldots \mu_{l}, \mu \nu}$ denote low-energy constants. The Latin indices run from 1 to 3 (only space derivatives), whereas the Greek indices run from 0 to 3 . The derivatives in the square brackets act only on the function within the brackets. Also, as a con- vention, the values $m, n=0$ correspond to no derivatives in Eq. (9).

The comparison of the two-point functions at $O(A)$, calculated in the different theories, leads to the matching condition

$$
\sum_{m, n=0}\left(-i p^{\prime}\right)_{i_{1}} \ldots\left(-i p^{\prime}\right)_{i_{n}}(i p)_{j_{1}} \ldots(i p)_{j_{m}} \Gamma_{s^{\prime} s}^{i_{1} \ldots i_{n}, j_{1} \ldots j_{m}, \mu}=\left\langle p^{\prime}, s^{\prime}\left|j^{\mu}(0)\right| p, s\right\rangle
$$

The expression of the second derivative of the two-point function with respect to the external field in the effective theory consists of two parts: the contribution from $\mathcal{L}_{2}$ and the nucleon pole term, denoted by $U^{\mu \nu}\left(p^{\prime}, s^{\prime} ; p, s ; q\right)$, which is obtained by the insertion of two vertices $\mathcal{L}_{1}$. The latter can be expressed through the nucleon vertex (an explicit expression is given below). The matching condition at $O\left(A^{2}\right)$ takes the form:

$$
\begin{aligned}
& \sum_{l, m, n=0}\left(-i p^{\prime}\right)_{i_{1}} \ldots\left(-i p^{\prime}\right)_{i_{n}}(i p)_{j_{1}} \ldots(i p)_{j_{m}}(i q)_{\mu_{1}} \ldots(i q)_{\mu_{l}} \Pi_{s^{\prime} s}^{i_{1} \ldots i_{n}, j_{1} \ldots j_{m}, \mu_{1} \ldots \mu_{l}, \mu \nu} \\
= & T^{\mu \nu}\left(p^{\prime}, s^{\prime} ; p, s ; q\right)-U^{\mu \nu}\left(p^{\prime}, s^{\prime} ; p, s ; q\right) .
\end{aligned}
$$

As can be seen, the low-energy constants of the effec- 
sion of the nucleon vertex and the Compton scattering amplitude in the external three-momenta.

\section{ENERGY SHIFT}

Up to now, we have considered the problem in a generic external field. We next limit ourselves to a static periodic magnetic field of the form

$$
\mathbf{B}=\left(0,0, B_{3}\right), \quad B_{3}=-e B \cos \left(\omega x_{2}\right),
$$

where $B$ denotes the strength of the field and the real parameter $\omega$ takes nonzero values. The components of the gauge field $A^{\mu}(x)$ read

$$
A_{1}=\frac{e B}{\omega} \sin \left(\omega x_{2}\right), \quad A_{0}=A_{2}=A_{3}=0 .
$$

The parameter $\omega$ allows one to scan the virtuality of the photon.

Since lattice simulations are performed in a finite spatial volume, the magnetic flux is quantized [37]. Consequently, the parameter $\omega$ can take only particular values (see, e.g., Refs. [38, 39]),

$$
\omega=\frac{2 \pi N}{L}, \quad N \in \mathbb{Z} \backslash\{0\},
$$

whereas the field strength $B$ is not quantized. Here, $L$ denotes the spatial size of the lattice. The quantization condition Eq. (14) also guaranties the proper implementation of the magnetic field on a torus [38]. We note that, for $\omega \neq 0$, there exists an alternative procedure that implies the quantization of the field strength $B$ instead of $\omega$ [38. In the present paper, however, we do not consider this option.

The quantum-mechanical Hamiltonian, which acts on the single-nucleon wave function as a differential operator, can be straightforwardly derived from the effective Lagrangian, Eq. (7). It is convenient to first rescale the nucleon field

$$
\psi(\mathbf{x}, t) \rightarrow \frac{1}{\sqrt{2 W(\nabla)}} \psi(\mathbf{x}, t) .
$$

The terms in the Hamiltonian can be again ordered, according to the powers of $A_{\mu}$ :

$$
H=H_{0}+H_{1}+H_{2}+O\left(A^{3}\right) .
$$

For instance, the free Hamiltonian reads $H_{0}=W(\nabla) \delta_{s^{\prime} s}$, and so on. To the best of our knowledge, an analytic solution of the Schrödinger equation in the periodic magnetic field is not available in the literature. For this reason, we resort to perturbation theory. The solutions must obey periodic boundary conditions. Denoting the Hilbert-state vector in the nonrelativistic theory, corresponding to the unperturbed solution, by $\left.\left|\mathbf{k}_{n}, s\right\rangle\right\rangle$, one has

$$
\left\langle\left\langle\mathbf{x} \mid \mathbf{k}_{n}, s\right\rangle\right\rangle=\frac{1}{L^{3 / 2}} e^{i \mathbf{k}_{n} \mathbf{x}} \chi_{s} .
$$

where $\chi_{s}$ denotes a Pauli spinor and

$$
w\left(\mathbf{k}_{n}\right)=\sqrt{m^{2}+\mathbf{k}_{n}^{2}}, \quad \mathbf{k}_{n}=\frac{2 \pi \mathbf{n}}{L}, \quad \mathbf{n} \in \mathbb{Z}^{3} .
$$

These vectors are normalized, according to $\left\langle\left\langle\mathbf{k}_{m}, s^{\prime} \mid \mathbf{k}_{n}, s\right\rangle\right\rangle=\delta_{\mathbf{m n}} \delta_{s^{\prime} s}$.

Next, we evaluate the energy shift of the nucleon ground state up to order $A^{2}$. Since the unperturbed solution is twofold degenerate due to the nucleon spin, one should use perturbation theory for the degenerate states. First, one may check that the first-order energy shift vanishes identically for $\omega \neq 0$, since, in this case, $\left\langle\left\langle\mathbf{0}, s\left|H_{1}\right| \mathbf{0}, s^{\prime}\right\rangle\right\rangle=0$ due to three-momentum conservation. Further, in order to determine the spin-averaged shift $\delta E$ at second order, the calculation of the diagonal matrix elements suffices:

$$
\delta E=\frac{1}{2} \sum_{s}\left(\delta E_{s}^{\prime}+\delta E_{s}{ }^{\prime \prime}\right),
$$

where the first contribution (the nucleon pole term) emerges from the second iteration of $H_{1}$ and the second term is the matrix element of $H_{2}$. The explicit expressions are

$$
\begin{aligned}
\delta E_{s}^{\prime} & =\sum_{\mathbf{k}_{n} \neq \mathbf{0}} \sum_{\sigma} \frac{\left.\left\langle\left\langle\mathbf{0}, s\left|H_{1}\right| \mathbf{k}_{n}, \sigma\right\rangle\right\rangle\left\langle\mathbf{k}_{n}, \sigma\left|H_{1}\right| \mathbf{0}, s\right\rangle\right\rangle}{w(\mathbf{0})-w\left(\mathbf{k}_{n}\right)}, \\
\delta E_{s}^{\prime \prime} & =\left\langle\left\langle\mathbf{0}, s\left|H_{2}\right| \mathbf{0}, s\right\rangle\right\rangle .
\end{aligned}
$$

The calculation of these corrections is straightforward and we obtain

$$
\begin{aligned}
\delta E_{s}^{\prime} & =\frac{(e B)^{2}}{8 m \omega^{2}}[F(\boldsymbol{\omega})+F(-\boldsymbol{\omega})], \\
\delta E_{s}^{\prime \prime} & =-\frac{(e B)^{2}}{4 m \omega^{2}}\left[T^{11}(0, s ; 0, s ; \hat{q})-U^{11}(0, s ; 0, s ; \hat{q})\right],
\end{aligned}
$$

where $\boldsymbol{\omega}=(0, \omega, 0), \hat{q}=(0, \boldsymbol{\omega})$ and

$$
F(\boldsymbol{\omega})=\sum_{\sigma} \frac{\left\langle\mathbf{0}, s\left|j^{1}(0)\right| \boldsymbol{\omega}, \sigma\right\rangle\left\langle\boldsymbol{\omega}, \sigma\left|j^{1}(0)\right| \mathbf{0}, s\right\rangle}{2 w(\boldsymbol{\omega})(w(\mathbf{0})-w(\boldsymbol{\omega}))} .
$$

The crucial step in getting these formulas has been to apply the matching conditions Eqs. (10) and (11). Note also that here, having used the matching condition, we switched back to the relativistic normalization of the state vectors.

It remains to calculate the quantity $U^{11}(0, s ; 0, s ; \hat{q})$. Inserting a complete set of the one-nucleon states into the pertinent matrix element and using the matching condition Eq. (10) once more, we obtain

$$
U^{11}(0, s ; 0, s ; \hat{q})=-\frac{1}{2}[F(\boldsymbol{\omega})+F(-\boldsymbol{\omega})] .
$$

Finally, adding everything together, we get a remarkably simple formula for the spin-averaged energy shift

$$
\begin{aligned}
\delta E & =\frac{(e B)^{2}}{4 m} T_{1}\left(0,-\omega^{2}\right)+O\left(B^{3}\right) \\
& =\frac{(e B)^{2}}{4 m} S_{1}\left(-\omega^{2}\right)+O\left(B^{3}\right)
\end{aligned}
$$


where the expression for the spin-averaged tensor Eq. (3) has been explicitly used. This is the main result of the present work. Note that the quantity $S_{1}$ here is the full one and not the inelastic part only.

\section{DISCUSSION AND CONCLUSIONS}

The applicability of the formula Eq. (24) is limited for several reasons. First, the strength of the magnetic field has to be chosen sufficiently small, so that perturbation theory provides a meaningful result. A necessary condition for this is that the structure of the perturbed and the unperturbed spectrum remains the same.

An estimate for the upper bound on the magnitude of $B$ can be obtained as follows. It can be straightforwardly checked that using the periodic external field, Eq. (13), in the Schrödinger equation leads to a periodic potential with magnitude $V_{0}=e^{2} B^{2} /\left(2 m \omega^{2}\right)$ and period $d=\omega^{-1}$. Considering a single period as a potential well, perturbation theory is applicable, if the well is shallow enough, so that no bound states are formed (in the periodic potential, the band structure arises instead of the isolated energy levels). Using, for simplicity, the known formula for the square well gives the condition $e B<2 \omega^{2}$. Of course, this should only be considered as a crude orderof-magnitude estimate of the critical value of $B$. Note also that, from the point of view of phenomenological applications, the region of the small $\omega^{2}$ (smaller than a few $\mathrm{GeV}^{2}$ ) is the most relevant one.

As seen from Eq. (24), the perturbative result is valid up to terms of order $B^{3}$. Albeit, in principle, it is possible to give some crude estimate of the neglected terms by using ChPT, it is important to note that the validity of the formula can be checked a posteriori on the lattice-by ensuring that the energy shift grows quadratically with $B$.

On the other hand, the value of $B$ should be large enough so that the energy shift $\delta E$ is measurable. Moreover, the accuracy of the extraction should be sufficient to allow for a disentanglement of the inelastic and elastic contributions. The latter is given by [4]

$$
S_{1}^{e l}\left(q^{2}\right)=-\frac{4 m^{2}}{q^{2}\left(4 m^{2}-q^{2}\right)}\left\{G_{E}^{2}\left(q^{2}\right)-G_{M}^{2}\left(q^{2}\right)\right\},
$$

where $G_{E}, G_{M}$ denote the electric and magnetic form factors, respectively. Further, the inelastic contribution at $q^{2}=0$ is related to the magnetic polarizability by [4]

$$
S_{1}^{i n e l}(0)=-\frac{\kappa^{2}}{4 m^{2}}-\frac{m}{\alpha} \beta_{M}
$$

where $\kappa$ denotes the anomalous magnetic moment of a nucleon and $\alpha$ is the fine-structure constant. Experimental values for the proton and neutron polarizabilities are $\beta_{M}^{p}=(3.15 \pm 0.50) \cdot 10^{-4} \mathrm{fm}^{3}$ [40] and $\beta_{M}^{n}=$
$(3.65 \pm 1.50) \cdot 10^{-4} \mathrm{fm}^{3}$ [41], respectively. As already mentioned, little is known about the $q^{2}$ dependence of $S_{1}\left(q^{2}\right)$. For a crude estimate, however, we assume that [4]

$$
S_{1}^{\text {inel }}\left(q^{2}\right)=\frac{S_{1}^{\text {inel }}(0)}{\left(1-q^{2} / 0.71 \mathrm{GeV}^{2}\right)^{2}} .
$$

Using now Eq. (24), it is immediately seen that the inelastic shift $\delta E^{\text {inel }}$ obeys the following relation:

$$
e B=\left(\frac{4 m \delta E^{\text {inel }}}{S_{1}^{\text {inel }}\left(q^{2}\right)}\right)^{1 / 2}=c \times\left|\delta E^{\text {inel }}\right|^{1 / 2}
$$

Taking now $-q^{2}=\omega_{\max }^{2}=2 \mathrm{GeV}^{2}$ as the upper bound of the interval, we get a crude estimate of the coefficient $c=2.97 \mathrm{GeV}^{3 / 2}$ for the proton and $c=2.77 \mathrm{GeV}^{3 / 2}$ for the neutron. Note that, using a rather generous estimate $\left|\delta E^{\text {inel }}\right|=0.05 \mathrm{~m}$, the bound Eq. (28) at $-q^{2}=\omega^{2}$ is comfortably consistent with the upper bound $e B<2 \omega^{2}$, except very small values of $\omega^{2}$.

The total energy shift $\delta E=\delta E^{e l}+\delta E^{\text {inel }}$ can be much larger than the inelastic shift alone, especially as $\omega^{2}$ is small. As seen from Eq. (25), the elastic contribution is singular as $\omega^{2} \rightarrow 0$, whereas the inelastic contribution is regular. As an order-of-magnitude estimate, one may ask, at what value of $\omega^{2}$ the magnitude of the inelastic contribution amounts to a $10 \%$ of the singular piece of the elastic contribution, which behaves like $1 / \omega^{2}$. The estimate gives $\omega_{\text {min }}^{2}=0.11 \mathrm{GeV}^{2}$ for the proton and $\omega_{\text {min }}^{2}=0.05 \mathrm{GeV}^{2}$ for the neutron. Of course, setting a lower bound on the $\omega^{2}$-interval critically depends on our ability to extract the proton and neutron form factors on the lattice with high accuracy. Note also that, even for the lowest value of $\omega_{\text {min }}^{2}$, the quantity $M_{\pi} L$ is of order of 4 -in other words, the calculations can be performed at the volumes which are feasible at present (here, $M_{\pi}$ is the pion mass).

Another interesting issue is the study of the limit $\omega \rightarrow 0$. In the present framework, this limit is singular and is intertwined with the limit $L \rightarrow \infty$. Indeed, recall that the values of $\omega$ are quantized: $\omega=2 \pi N / L$. For nonzero values of $N$, the limit $\omega \rightarrow 0$ thus implies $L \rightarrow \infty$. In addition, the Landau levels are bound even if $L \rightarrow \infty$, which violates the condition of the weak $B$ field. We expect that the alternative setting for the periodic magnetic field on the lattice (quantized $B, \omega$ not quantized), see Ref. 38], which has not been considered in the present paper, will be more advantageous for studying the limit $\omega \rightarrow 0$. Also, this different approach will allow one to continuously scan the interval of interest in the variable $q^{2}$. We plan to address these and other issues in a forthcoming work [34].

To summarize, our final expression, Eq. (24), enables one to directly extract the subtraction function $S_{1}\left(q^{2}\right)$ from the lattice measurement of the nucleon energy levels in a periodic external magnetic field. 


\section{ACKNOWLEDGMENTS}

We thank Z. Davoudi, J. Gasser, H. Leutwyler, J. A. Oller, M. Savage, G. Schierholz and N. Tantalo for useful discussions. We acknowledge the support from the DFG (CRC 110 "Symmetries and the Emergence of Structure in QCD" and Bonn-Cologne Graduate School of Physics and Astronomy). This research is supported in part by Volkswagenstiftung under Contract No. 86260 and by the Chinese Academy of Sciences (CAS) President's International Fellowship Initiative (PIFI) (Grant No. 2017VMA0025).

[1] S. Borsanyi et al., Science 347, 1452 (2015).

[2] R. Horsley et al., J. Phys. G 43, 10LT02 (2016).

[3] J. Gasser and H. Leutwyler, Nucl. Phys. B 94, 269 (1975).

[4] J. Gasser, M. Hoferichter, H. Leutwyler and A. Rusetsky, Eur. Phys. J. C 75, 375 (2015).

[5] A. Walker-Loud, C. E. Carlson and G. A. Miller, Phys. Rev. Lett. 108, 232301 (2012).

[6] F. B. Erben, P. E. Shanahan, A. W. Thomas and R. D. Young, Phys. Rev. C 90, 065205 (2014).

[7] M. C. Birse and J. A. McGovern, Eur. Phys. J. A 48, 120 (2012).

[8] K. Pachucki, Phys. Rev. A 60, 3593 (1999).

[9] C. Peset and A. Pineda, Nucl. Phys. B 887, 69 (2014)

[10] J. C. Collins, Nucl. Phys. B 149, 90 (1979); B 915, 392 (2017).

[11] R. J. Hill and G. Paz, arXiv:1611.09917.

[12] V. Bernard, N. Kaiser and U.-G. Meißner, Nucl. Phys. B 373, 346 (1992).

[13] V. Bernard, N. Kaiser, J. Kambor and U.-G. Meißner, Nucl. Phys. B 388, 315 (1992).

[14] X. D. Ji, C. W. Kao and J. Osborne, Phys. Rev. D 61, 074003 (2000).

[15] V. Bernard, T. R. Hemmert and U.-G. Meißner, Phys. Rev. D 67, 076008 (2003).

[16] R. J. Hill and G. Paz, Phys. Rev. Lett. 107, 160402 (2011).

[17] J. M. Alarcon, V. Lensky and V. Pascalutsa, Eur. Phys. J. C 74, 2852 (2014).
[18] V. Lensky, J. M. Alarcon and V. Pascalutsa, Phys. Rev. C 90, 055202 (2014).

[19] D. Nevado and A. Pineda, Phys. Rev. C 77, 035202 (2008).

[20] M. Gorchtein, F. J. Llanes-Estrada and A. P. Szczepaniak, Phys. Rev. A 87, 052501 (2013).

[21] O. Tomalak and M. Vanderhaeghen, Eur. Phys. J. C 76, 125 (2016).

[22] S. J. Brodsky, F. J. Llanes-Estrada and A. P. Szczepaniak, Phys. Rev. D 79, 033012 (2009).

[23] J. Green, O. Gryniuk, G. von Hippel, H. B. Meyer, and V. Pascalutsa, Phys. Rev. Lett. 115, 222003 (2015).

[24] N. H. Christ, X. Feng, A. Portelli, and C. T. Sachrajda (RBC and UKQCD Collaborations), Phys. Rev. D 93, 114517 (2016).

[25] N. H. Christ, X. Feng, A. Jüttner, A. Lawson, A. Portelli, and C. T. Sachrajda, Phys. Rev. D 94, 114516 (2016).

[26] B. C. Tiburzi and S. O. Vayl, Phys. Rev. D 87, 054507 (2013).

[27] E. Chang, W. Detmold, K. Orginos, A. Parreño, M. J. Savage, B. C. Tiburzi, and S. R. Beane (NPLQCD Collaboration), Phys. Rev. D 92, 114502 (2015).

[28] A. Parreño et al., arXiv:1609.03985 [hep-lat].

[29] W. Detmold, B. C. Tiburzi and A. Walker-Loud, Phys. Rev. D 73, 114505 (2006).

[30] Z. Davoudi and W. Detmold, Phys. Rev. D 93, 014509 (2016).

[31] C. Kittel, Introduction to Solid State Physics, 7th ed. (Wiley, New York, 1996).

[32] R. Tarrach, Nuovo Cimento Soc. Ital. Fis., A 28, 409 (1975).

[33] J. Bernabeu and R. Tarrach, Ann. Phys. (N.Y.) 102 (1976) 323.

[34] A. Agadjanov, U.-G. Meißner and A. Rusetsky (to be published)

[35] G. Colangelo, J. Gasser, B. Kubis and A. Rusetsky, Phys. Lett. B 638, 187 (2006).

[36] J. Gasser, B. Kubis and A. Rusetsky, Nucl. Phys. B 850, 96 (2011).

[37] G. 't Hooft, Nucl. Phys. B 153, 141 (1979).

[38] Z. Davoudi and W. Detmold, Phys. Rev. D 92, 074506 (2015).

[39] G. Bali and G. Endrödi, Phys. Rev. D 92, 054506 (2015).

[40] J. A. McGovern, D. R. Phillips and H. W. Grießhammer, Eur. Phys. J. A 49, 12 (2013).

[41] L. S. Myers et al. [COMPTON@MAX-lab Collaboration], Phys. Rev. Lett. 113, 262506 (2014). 